\title{
Influência do treinamento de Karate-Dō na cognição de idosos com Transtorno Neurocognitivo Leve: Ensaio clínico aberto
}

\author{
Influence of Karate-Do training in the cognition of old people with Mild Cognitive Impairments:
} Open study

\section{Influencia del entrenamiento de Kárate en la cognición de Mayores con Trastorno Neurocognitivo}

\author{
Ligero: Ensayo clínico abierto
}

Recebido: 11/06/2021 | Revisado: 18/06/2021 | Aceito: 21/06/2021 | Publicado: 05/07/2021

\author{
Brandel José Pacheco Lopes Filho \\ ORCID: https://orcid.org/0000-0001-6666-9637 \\ Universidade do Estado de Minas Gerais, Brasil \\ E-mail: brandelfilho@gmail.com \\ Camila Rosa de Oliveira \\ ORCID: https://orcid.org/0000-0003-2115-604X \\ Faculdade Meridional IMED, Brasil \\ E-mail: oliveira.crd@gmail.com \\ Maria Gabriela Valle Gottlieb \\ ORCID: https://orcid.org/0000-0002-7694-0336 \\ Pontifícia Universidade Católica do Rio Grande do Sul, Brasil \\ E-mail: vallegot@hotmail.com
}

\begin{abstract}
Resumo
Artes marciais são consideradas formas de exercício completas e podem contribuir para estimular habilidades motoras e cognitivas. O objetivo deste trabalho foi verificar a eficácia do treinamento de Karate-Dō, estilo Wadō-ry $\bar{u}$, na cognição de idosos diagnosticados com transtorno neurocognitivo leve. Realizou-se, portanto, um ensaio clínico com oito idosos, que responderam a uma bateria de testes cognitivos. A análise dos dados foi realizada com estatísticas descritivas e inferenciais. Identificou-se que no período pós-intervenção o grupo obteve pontuação significativamente maior nas tarefas de Dígitos - ordem indireta $(p=0,023)$, RAVLT A1 a A5 $(p=0,018)$ e Reconhecimento $(p=0,011)$, WCST - Respostas de nível conceitual $(p=0,020)$ e Categorias completas $(p=, 034)$, Figura Complexa de Rey Evocação $(p=0,027)$ e AMV ordem direta $(p=0,017)$ e indireta $(p=0,033)$. Na etapa pré intervenção, o grupo apresentou maior tempo na realização da tarefa TMT-B, havendo redução significativa $(p=0,012)$ neste tempo após o período de treinamento, indicando uma melhora na habilidade de atenção alternada. Os resultados sugerem que a prática de três meses de Karate-Dō, estilo Wadō-ryū, está relacionada a uma melhora significativa da cognição nos aspectos de memória visual, atenção, atenção alternada, memória de trabalho, memória verbal episódica e funções executivas.
\end{abstract}

Palavras-chave: Envelhecimento; Artes marciais; Karate; Cognição; Ensaio clínico.

\begin{abstract}
Martial arts are considered complete forms of exercise and can contribute to stimulating motor and cognitive skills. The aim of this study was to verify the effectiveness of Wado-ryu Karate-Do training on the cognition of old people diagnosed with mild neurocognitive impairment. Therefore, a clinical trial was carried out with eight people, who responded to a battery of cognitive tests. Data analysis was performed using descriptive and inferential statistics. It was found that in the post-intervention period, the group scored significantly higher on the Digits tasks - forward $(p=$ $0.023)$, RAVLT A1 to A5 $(p=0.018)$ and Recognition $(p=0.011)$, WCST - Conceptual Level Responses $(p=0.020)$ and Complete Categories ( $p=.034)$, Rey's Complex Figure - delayed recall $(p=0.027)$ and Spatial Spam forward $(p$ $=0.017)$ and backward $(p=0.033)$. In the pre-intervention stage, the group had a longer time to perform the TMT-B task, with a significant reduction $(p=0.012)$ in their time after the intervention, indicating an improvement in the alternating attention skill. The results suggest that the three-month practice of Wado-ryu Karate-Do is related to a significant improvement in cognition tasks of visual memory, attention, alternate attention, working memory, episodic verbal memory and executive functions.
\end{abstract}

Keywords: Aging; Martial arts; Karate; Cognition; Clinical trial.

\section{Resumen}

Las artes marciales se consideran formas completas de ejercicio y pueden contribuir a estimular las habilidades motoras y cognitivas. El objetivo de este trabajo fue verificar la efectividad del entrenamiento de Kárate, estilo Wadō- 
ryū, sobre la cognición de personas mayores diagnosticadas con trastorno neurocognitivo ligero. Por tanto, se realizó un ensayo clínico con ocho personas, que respondieron a una batería de evaluación cognitiva. El análisis de los datos se realizó mediante estadística descriptiva y inferencial. Se encontró que, en el período posterior a la intervención, el grupo puntuó significativamente más alto en las tareas de Dígitos - orden indirecta $(p=0.023)$, RAVLT A1 a A5 ( $p=$ $0.018)$ y Reconocimiento $(p=0.011)$, WCST - Respuestas de categorías conceptual $(p=0.020)$ y Completa $(p=$ $.034)$, Figura compleja de Rey - Evocación $(p=0.027)$ y AMV orden directa $(p=0.017)$ e indirecta $(p=0.033)$. En la etapa de pre intervención, el grupo tuvo un mayor tiempo para realizar la tarea TMT-B, con una reducción significativa $(p=0.012)$ en este tiempo después del período de entrenamiento, lo que indica una mejora en la habilidad de atención alterna. Los resultados sugieren que la práctica de tres meses de Kárate, estilo Wadō-ryū, se relaciona con una mejora significativa en la cognición en tareas de memoria visual, atención, atención alterna, memoria de trabajo, memoria verbal episódica y funciones ejecutivas.

Palabras clave: Envejecimiento; Artes marciales; Kárate; Cognición; Ensayo clínico.

\section{Introdução}

Funções cognitivas são o conjunto de habilidades que tratam da capacidade cerebral de conhecer, interpretar e agir no meio em que o indivíduo está inserido, referindo-se a diferentes processos mentais: atenção, percepção, memória, linguagem, habilidades visuoconstrutivas e motoras, além das funções executivas (Sternberg, 2008). Ao longo do processo do envelhecimento, algumas funções apresentam declínio natural (Salthouse, 2014), sendo que a velocidade de tais perdas pode ser influenciada pelo estilo de vida do indivíduo, sendo a prática de exercícios físicos um fator protetivo (La Rue, 2010; Proust-Lima et al. 2008), podendo reduzir o impacto de processos neurodegenerativos decorrentes do envelhecimento (Northey, Cherbuin, Pumpa, Smee \& Rattray, 2018; Hillman et al., 2014; Stern, 2012; Nucci, Mapelli \& Mondini, 2012; Diamond \& Lee, 2011; Rami et al., 2011), especialmente quando tais práticas exigem maior envolvimento e atenção (Jäger, Schmidt, Conzelmann \& Roebers, 2015).

A prática de exercícios físicos influencia positivamente no desempenho cognitivo (La Rue, 2010) e, de acordo com Nunan (2006), artes marciais são formas de exercício completas, que trazem benefícios à saúde física, além de contribuir para estimular habilidades motoras e cognitivas, melhorar a qualidade de vida e aspectos emocionais (Jansen \& Dahmen-Zimmer, 2012; Burke, Al-Adawi, Lee \& Audette, 2007). O Karate-D $\bar{o}^{1}$, enquanto arte marcial, além de trabalhar o corpo, estimula a memória e o aprendizado de um conjunto de habilidades variadas. O seu ensino é alicerçado em três pilares pedagógicos centrais: kihon (fundamento), kata (forma) e kumite (luta, combate) (Funakoshi, 1973). Kihon é a prática dos fundamentos, como socos, chutes e bloqueios, no qual o praticante repete os movimentos em busca do aprimoramento dos gestos técnicos, individualmente. Os Kata se constituem em sequências pré-determinadas de movimentos, realizadas de forma individual, que simulam um combate contra múltiplos adversários. Por último, o Kumite trata de exercícios reais ou adaptados de combate corporal entre dois ou mais praticantes (Nakayama, 2000a; 2000b). Trata-se de um processo de desenvolvimento global, no qual o foco do karateka é voltado para a técnica e coordenação corporal, o que indica a presença de uma exigente tarefa cognitiva, aliada ao trabalho de memória muscular e práticas de meditação e concentração, aumentando a tranquilidade e beneficiando o equilíbrio psicológico (Jansen \& Dahmen-Zimmer, 2012).

Diversos trabalhos apontam benefícios para estas funções, decorrentes da prática específica de artes marciais, especialmente em tarefas de atenção (Lopes Filho, 2015), memória funcional (Man, Tsang, \& Hui-Chan, 2010), função cognitiva global, memória tardia e queixas cognitivas subjetivas (Lam et al., 2011), além do desempenho cognitivo geral (Nguyen \& Kruse, 2012), processamento de informação, planejamento e emissão de respostas motoras a estímulos (Lima et al., 2017). No entanto, há uma grande carência de investigações dos efeitos terapêuticos do Karate-Dō quanto aos aspectos cognitivos, ainda mais no caso de adultos idosos (Lopes Filho, Oliveira \& Gottlieb, 2019), sendo essencial entender os efeitos

\footnotetext{
1 Para a escrita de palavras japonesas e da língua nativa de Okinawa (Uchināguchi), adotou-se a romanização padronizada pelo sistema Hepburn (ヘボン), seguindo as normativas internacionais.
} 
dessa atividade para possível uso em técnicas de intervenção em quadros neurológicos, através de exercício/práticas esportivas. Com base nisso, o objetivo deste estudo foi verificar a eficácia do treinamento de Karate-Dō estilo Wad̄o-ryū na cognição de idosos diagnosticados com transtorno neurocognitivo leve.

\section{Método}

Este estudo é caracterizado como um ensaio clínico do tipo aberto, método com aplicação de grupos de intervenção com seres humanos, sem mascaramento, nos quais todos os envolvidos sabem a que grupo pertence cada indivíduo da casuística (Hochman, Nahas, Oliveira Filho \& Ferreira, 2005; Greenhalgh, 2005). Foi aprovado pelo Comitê de Ética em Pesquisa da Pontifícia Universidade Católica do Rio Grande do Sul (CAAE $n^{\circ}$ 26742314.8.0000.5336) e catalogado junto ao Registro Brasileiro de Ensaios Clínicos (REBEC n² 2494). As exigências da Resolução 466/2012 do Ministério da Saúde sobre Pesquisa envolvendo seres humanos foram cumpridas.

\subsection{Amostra}

Participaram, ao todo, oito idosos com idade $\geq 60$ anos, residentes do Município de Porto Alegre. Eles foram recrutados por conveniência, provenientes de um ensaio clínico maior, que avaliou a capacidade de idosos saudáveis quanto aos aspectos cognitivos, sendo escolhidos para este recorte os que apresentaram diagnóstico de transtorno neurocognitivo leve.

Como critérios de inclusão foram assumidos: ser alfabetizado, ter idade $\geq 60$ anos; apresentarem atestado médico autorizando-os a praticar exercícios; apresentar diagnóstico de transtorno neurocognitivo leve (na fase de testes préintervenção); assinarem o termo de consentimento livre e esclarecido. Os critérios de exclusão foram: realizar exercício físico de forma regular; utilizar medicamento(s) antidepressivo(s); ser alcoolista; apresentar pontuação abaixo do ponto de corte do Mini Exame do Estado Mental (MEEM, adaptado por Chaves \& Izquierdo, 1992); autorrelato de dificuldades de locomoção para realização da prática de intervenção, ou que requeiram uso de acessórios para auxiliar no deslocamento.

\subsection{Bateria cognitiva}

Os idosos participaram, individualmente, de uma sessão de aproximadamente 90 minutos, na qual preencheram uma ficha de caracterização sociodemográfica e clínica e responderam à bateria cognitiva. Essa bateria foi reaplicada após o período de treinamento (12 semanas) para averiguar os resultados. Os testes foram conduzidos por uma psicóloga, em sala adequada localizada no Programa de Pós-Graduação em Psicologia da PUCRS, e pontuada por outro profissional da pesquisa, para evitar conflitos quanto à pontuação. Ambos receberam treinamento para aplicação e correção dos testes.

A bateria incluiu: (1) MEEM: avalia orientação para tempo, local, registro de três palavras, atenção e cálculo, lembrança de três palavras, linguagem e capacidade construtiva visual. Foi utilizada a versão em português (traduzida por Chaves \& Izquierdo, 1992); (2) Trail Making Test (TMT): fornece medidas de atenção dividida e velocidade de processamento visual (Army Individual Test Battery, 1944); (3) Subteste Dígitos da Escala Wechsler de Inteligência para Adultos, terceira edição: avalia atenção concentrada e memória de trabalho (adaptado por Nascimento, 2004); (4) Figura Complexa de Rey (FCR): avalia percepção visual, funções visuoconstrutivas, capacidade de planejamento e memória visual de evocação tardia (adaptado por Oliveira \& Rigoni, 2010); (5) Rey Auditory-Verbal Learning Test (RAVLT): mensura memória verbal episódica (modalidades evocação imediata - RAVLT A1-A5; e tardia - RAVLT A7) e memória de reconhecimento (RAVLT reconhecimento) (adaptado por Malloy-Diniz, Lasmar, Gazinelli, Fuentes \& Salgado, 2007); (6) Subteste Amplitudade da Memória Visual (AMV) da Escala Wechsler de Memória: avalia atenção concentrada (modalidade visual) e memória visuoespacial (Wechsler, 1987); (7) Tarefa Motora Sequencial (Praxia Ideomotora de Luria): avalia capacidade de sequenciamento motor através de movimentos com a mão (pontuação sugerida por Nitrini, Caramelli, Herrera Jr., Charchat- 
Fichman \& Porto, 2005); (8) Teste Wisconsin de Classificação de Cartas (WCST-64): avalia as funções executivas e requer a capacidade de desenvolver e manter uma estratégia apropriada para a resolução dos problemas propostos (Cunha et al., 2005); (9) Fluência Verbal (FV - FAS e animais): investiga flexibilidade cognitiva, busca lexical e memória semântica (Strauss, Sherman \& Spreen, 2006); (10) Escala de Depressão Geriátrica (GDS-15): questionário breve (versão em português) para identificação e quantificação de sintomas depressivos em idosos (Almeida \& Almeida, 1999).

O diagnóstico de transtorno neurocognitivo leve foi realizado na coleta de dados inicial, por meio dos critérios do Manual Diagnóstico e Estatístico de Transtornos Mentais, quinta edição (American Psychiatric Association, 2014). São eles: (a) ocorrência de queixas cognitivas subjetivas (verificadas por meio de autorrelato na anamnese); (b) desempenho cognitivo deficitário nos testes cognitivos utilizados (escore $Z \leq-1,5$ ); e (c) capacidade funcional preservada, avaliada por meio de autorrelato na anamnese.

\subsection{Intervenção}

Após a avaliação inicial, o grupo participou de 24 sessões de intervenção ao longo de 12 semanas, realizadas em grupo, duas vezes por semana, com duração de 60 minutos. Os exercícios trabalhados foram: (1) preparação para o início da aula através de um breve aquecimento - 5 a 10 minutos; (2) exercícios de kihon (técnicas básicas), kata (sequências de movimentos pré-determinadas), kumite (exercícios de luta) e de respiração - 40 a 45 minutos; e (3) relaxamento/volta à calma através de exercícios de meditação breve adaptada - 10 minutos.

Os exercícios técnicos de Karate-Dō abordados nas sessões de treinamento foram os mesmos que constam no quadro de técnicas da primeira faixa (branca ou Mu Kyū), do estilo Wadō-ryū. No kihon: Jun Zuki (soco reto), Gyaku Zuki (soco invertido), Gedan Barai Uke (defesa baixa), Jodan Age Uke (defesa alta), Mae Geri (chute frontal) e Zenkutsu Dachi (base avançada); no kata: Kihon Kata (forma fundamental); e no kumite, aplicações em dupla dos exercícios supracitados. Exercícios de respiração foram inseridos dentro do próprio kihon. A prática de meditação foi realizada na posição decúbito dorsal, sobre colchonetes, de forma a evitar desconforto nas costas ou membros inferiores.

As práticas ocorreram em uma sala apropriada, climatizada e com piso emborrachado. Durante o período da intervenção os idosos foram instruídos a continuar realizando suas atividades cotidianas. Após o encerramento de todas as sessões, todos foram reavaliados com os mesmos instrumentos utilizados anteriormente. A figura 1 expõe o desenho do estudo e todas as suas etapas. Cabe salientar que os participantes excluídos deste recorte $(n=65)$ foram encaminhados para outra frente do estudo, cuja finalidade é diversa. Foram investigadas as variáveis: (1) cognição e (2) dados sociodemográficos. Com exceção do professor de Karate- $D \bar{o}$ e dos idosos treinados, todos os demais envolvidos no estudo foram cegados para sua realização. 
Figura 1. Fluxograma do ensaio clínico realizado.

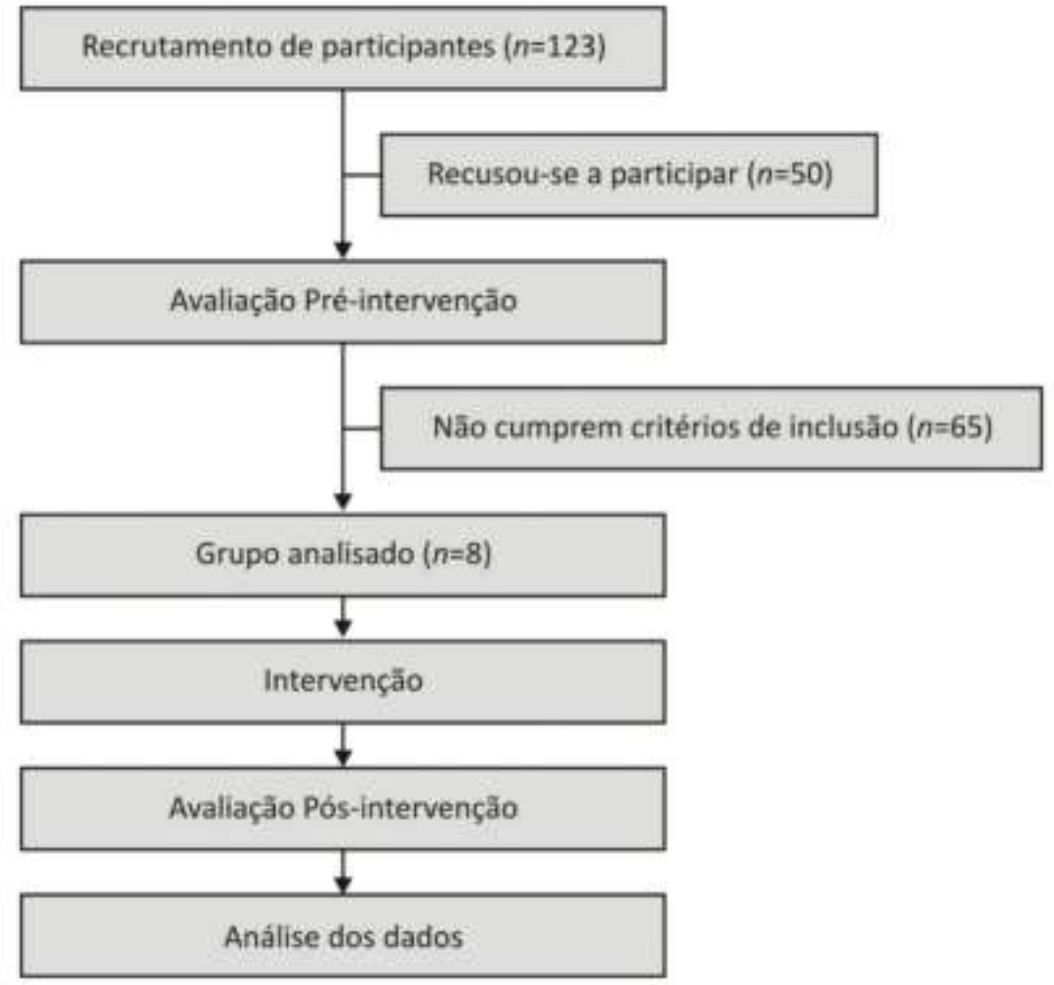

Fonte: Autores.

\subsection{Análise dos dados}

Os dados foram analisados através do pacote estatístico SPSS versão 23.0. A análise descritiva contemplou frequências, médias e desvios-padrão. O desempenho nos testes cognitivos foi analisado por meio do teste de Wilcoxon. Resultados significativos foram considerados quando $p \leq 0,05$.

\section{Resultados}

A amostra investigada foi composta por sete mulheres (87,5\%) e um homem (12,5\%), com idade média de 73,75 anos ( $\mathrm{DP}=5,80)$ e média de escolaridade em 13,13 ( $\mathrm{DP}=5,79)$ anos de estudo. A amostra não apresentou sintomas depressivos significativos. A Tabela 1 apresenta o desempenho do grupo na bateria de avaliação cognitiva, comparando os resultados pré e pós-intervenção.

Pode-se identificar que no período pós-intervenção o grupo obteve pontuação significativamente maior nas tarefas de Dígitos (ordem indireta), RAVLT (A1 a A5 e Reconhecimento), WCST (Respostas de nível conceitual; Categorias completas), Figura Complexa de Rey (evocação) e AMV (ordem direta e indireta), evidenciando um desempenho final superior ao compararmos com os dados da fase pré-intervenção. Na etapa pré intervenção, o grupo apresentou maior tempo na realização da tarefa TMT-B, havendo redução significativa neste tempo após o período de treinamento, indicando uma melhora na habilidade de atenção alternada. 
Tabela 1. Comparação do desempenho cognitivo pré e pós intervenção.

\begin{tabular}{|c|c|c|c|c|c|}
\hline & \multicolumn{2}{|c|}{ Pré } & \multicolumn{2}{|c|}{ Pós } & \multirow{2}{*}{$p$} \\
\hline & $\mathrm{M}$ & DP & $\mathrm{M}$ & DP & \\
\hline MEEM & 26,63 & 2,62 & 26,75 & 2,38 & 0,715 \\
\hline Dígitos - Ordem Direta & 6,50 & 1,77 & 6,75 & 0,89 & 0,683 \\
\hline Dígitos - Ordem Indireta & 3,75 & 1,49 & 5,00 & 0,93 & $\mathbf{0 , 0 2 3}$ \\
\hline Tempo(s) TMT-A & 55,00 & 22,69 & 51,07 & 21,73 & 0,093 \\
\hline Tempo(s) TMT-B & 211,78 & 91,45 & 163,60 & 79,96 & 0,012 \\
\hline RAVLT A1 até A5 & 39,38 & 8,02 & 45,13 & 8,68 & 0,018 \\
\hline RAVLT A7 & 7,38 & 1,92 & 7,88 & 3,23 & 0,598 \\
\hline RAVLT Reconhecimento & 5,00 & 5,15 & 8,00 & 4,38 & 0,011 \\
\hline WCST Erros perseverativos & 24,00 & 14,67 & 14,63 & 3,96 & 0,108 \\
\hline WCST Erros não perseverativos & 14,13 & 12,26 & 12,75 & 3,20 & 0,888 \\
\hline WCST Respostas de nível conceitual & 13,75 & 10,28 & 29,38 & 7,03 & $\mathbf{0 , 0 2 0}$ \\
\hline WCST Categorias completas & 1,00 & 0,93 & 2,13 & 0,83 & $\mathbf{0 , 0 3 4}$ \\
\hline WCST Rupturas & 0,13 & 0,35 & 0,38 & 0,74 & 0,414 \\
\hline Fluência Verbal FAS & 40,50 & 9,26 & 40,63 & 13,21 & 0,833 \\
\hline Figura Complexa de Rey (cópia) & 26,13 & 5,81 & 26,25 & 3,41 & 0,833 \\
\hline Fluência Verbal Animais & 16,88 & 4,39 & 19,50 & 6,14 & 0,088 \\
\hline Figura Complexa de Rey (evocação) & 10,63 & 5,97 & 13,75 & 4,18 & 0,027 \\
\hline AMV - Ordem Direta & 5,13 & 1,55 & 7,00 & 1,41 & 0,017 \\
\hline AMV - Ordem Indireta & 4,75 & 2,05 & 6,38 & 1,30 & $\mathbf{0 , 0 3 3}$ \\
\hline Praxia Idemotora de Luria & 1,13 & 2,10 & 1,25 & 0,71 & 0,565 \\
\hline
\end{tabular}

Nota . TMT-A = Trail Making Test parte A; TMT-B = Trail Making Test parte B; RAVLT = Rey Auditory Verbal Learning Test; WCST = Teste Wisconsin de Classificação de Cartas, versão de 64 cartas; Figura Complexa de Rey; AMV = Amplitude da memória visual; grau de liberdade $(\mathrm{gl})=31$; Comparação entre períodos pré e pós intervenção realizada com teste de Wilcoxon. Fonte: Autores.

\section{Discussão}

O grupo investigado apresentou um desempenho superior, em comparação ao período pré-intervenção, em habilidade de memória visual de evocação tardia (evidenciado pelo teste da Figura Complexa de Rey), memória de trabalho visual e verbal (Dígitos - ordem indireta; AMV - ordem indireta) e memória episódica (RAVLT reconhecimento). Tais dados podem estar relacionados às características da prática de Karate-Dō; em uma aula padrão, o aluno memoriza os movimentos e correções executados pelo professor para, então, realizar as tarefas sequenciais. Iniciando de forma mecânica, essa repetição evolui até que o praticante conte com sua memória para realizar as técnicas (Nakayama, 2000a).

Constatou-se que ocorreram melhoras significativas quanto à função de atenção (AMV - ordem direta) e atenção alternada (TMT-B - Tempo em segundos). Este comportamento pode estar associado às técnicas de kata (forma) e kumite (luta). Além dos estímulos visuais providos pelo professor, no kata os praticantes, em exercício coletivo, devem executar movimentos em sequência, deslocando-se pelo ambiente em diferentes direções (Nakayama, 2000a; 2000c), atentos para realizá-los de forma correta e evitando o choque com seus colegas. No kumite é preciso dosar potência, velocidade e distância, exercitando-se em sincronia com o colega; além disso, os exercícios são alternados entre ataques e defesas, com uso variado de membros inferiores e superiores (Nakayama, 2000d). São tarefas de grande demanda atencional e de habilidade visuoespacial.

Também foram constatadas melhoras significativas quanto à memória verbal episódica de evocação imediata (tarefa RAVLT A1-A5), a qual pode estar relacionada ao convívio social que ocorre na atividade (Vance, Wadley, Ball, Roenker \& 
Rizzo, 2005), pois as aulas foram coletivas e a turma mista, sendo a conversa e interação sempre estimuladas nos horários de intervalo, entrada e saída da prática. Outro aspecto que pode ter influenciado neste quesito é o conteúdo verbal específico do Karate-Dō; para as técnicas e movimentações são utilizados os nomes originais japoneses, os quais devem ser aprendidos pelos alunos ao longo do tempo, o que foi constantemente abordado nas sessões de treino.

Quanto aos aspectos de funções executivas, os testes WCST-64 (respostas de nível conceitual e categorias completas) e TMT-B (Tempo em segundos) identificaram melhoras na capacidade de raciocínio lógico-abstrato e de velocidade de processamento visual. Os estímulos visuais e as demandas exigidas no treinamento de Karate-Dō podem servir de estímulo quanto ao processamento e memória visual, especialmente no kumite (combate). Neste tipo de exercício, além de realizar o movimento de forma correta, deve-se estar atento para executar ataques e defesas de forma efetiva (Nakayama, 2000d), sendo que essas tarefas trabalham a velocidade de reação, cujo um dos aspectos é o elemento visual (Schmidt \& Lee, 2013). Já a capacidade de raciocínio lógico-abstrato está fortemente associada ao exercício de kata (forma): configurado como uma sequência de movimentos pré-determinados, o praticante deve fazer os movimentos na ordem correta, procurando acertar seus “adversários” em pontos específicos, que devem ser visualizados mentalmente (Nakayama 2000a; 2000c). Nos exercícios de kihon (fundamento) e kumite (combate) existem demandas similares (Nakayama, 2000b; 2000d), pois é necessário escolher e executar os movimentos mais apropriados para cada exercício/prática. O uso de um processo cognitivo que envolve raciocínio lógico-abstrato aparenta ser muito presente nestas tarefas, os quais podem ser indicativos da melhora obtida.

Diversos estudos indicam melhoras significativas na cognição após períodos de treinamento físico. Frederiksen et al. (2015) investigaram os efeitos de exercício físico em uma população de 282 idosos no período de três anos, encontrando benefícios relativos a funções executivas e velocidade de processamento. Antunes, Santos, Heredia, Bueno, \& Mello (2001) ofereceram um programa de condicionamento físico aeróbio de seis meses, no qual identificaram melhoras significativas em habilidades de atenção, memória e agilidade motora. Uma revisão sistemática com metanálise multinível (Northley et al., 2018), que incluiu 39 estudos com intervenções variadas (exercícios aeróbicos, treinamento de resistência, treinamento multicomponente e Tàijí quán), demonstrou que o exercício físico melhorou a função cognitiva em pessoas com 50 anos ou mais, independentemente do domínio cognitivo testado ou do estado cognitivo dos participantes. Além disso, exercícios resistidos (classificação parcialmente apropriada ao Karate-Dō e outras artes marciais) costumam apresentar melhoras significativas quanto ao funcionamento cognitivo global, especialmente em funções de memória, funções executivas, atenção e velocidade de processamento (Lima, 2014).

Especificamente em artes marciais, Kasai et al. (2010) encontraram melhoras significativas sobre a memória geral em um estudo com 26 idosas após seis meses de treinamento de Tàijí quán. Outro estudo (Man, Tsang, \& Hui-Chan, 2010) apontou benefícios para atenção concentrada, memória verbal episódica, memória visual e memória visuoespacial em idosos, com treinamento na mesma arte marcial no período de 10 semanas. Diversos estudos sobre Tàijí quán evidenciam melhoras na função global cognitiva, memória tardia e queixas cognitivas subjetivas (Lam et al., 2011), resultados significativos quanto às funções executivas (Nguyen \& Kruse, 2012) e velocidade de processamento (Chang et al., 2011). Em recente revisão sistemática (Rios, Marks, Estevan \& Barnett, 2017) foram investigados 28 estudos sobre os efeitos positivos das artes marciais (Karate-Dō, Jūdō, Taekwondo, Wǔshù e Tàijí quán) sobre a cognição, sendo que a maioria relatou resultados significativos, independentemente da idade de período de início da prática, beneficiando o equilíbrio das funções cognitivas e atuando como possível fator protetivo.

Sobre Karate-Dō, especificamente, existem poucos estudos na área. O estudo de Lima et al. (2017) encontrou resultados significativos no processamento da informação, planejamento e emissão de respostas motoras a estímulos em crianças. Há indícios de melhoras, também, quanto à questão do autoconceito de competência cognitiva na infância (Conant, Morgan, Muzykewicz, Clark \& Thiele, 2008), apesar da arte verificada ter sido uma prática similar, nomeada de Kenpō 
Karate. Há, ainda, a aplicação de treinamento de Karate-Dō (estilo Shōtōkan-ryū) sem resultados positivos (Jansen \& Dahmen-Zimmer, 2012). O estudo mais aprofundado na população idosa, contudo, permanece sendo o de Lopes Filho, Oliveira e Gottlieb (2019), que faz parte deste projeto. Na ocasião, investigou-se os efeitos da prática aguda de Karate-Dō, estilo Wadō-ryū, por 12 semanas (duas sessões por semana, com uma hora de duração cada), constatando melhorias em habilidades de atenção, memória e funções executivas, além de queixas subjetivas relacionadas à memória.

A melhora cognitiva obtida através da prática do Karate-Dō, investigada pelo presente trabalho, pode ser explicada pela presença de um intenso treinamento de memória para aprender a sequência de tarefas realizadas nas sessões de treinamento. Trata-se de uma ação coordenada entre memória, atenção, ações motoras voluntárias e de imagem verbal e visual, os quais fazem parte do processo de aprendizagem (Lam et al., 2011). Além disso, o Tàijí quán é assumido como uma ferramenta eficaz para prevenir o declínio cognitivo causado pelo envelhecimento (normal ou patológico), pois sua prática estimula os processos de neurogênese e angiogênese no cérebro (Chang et al., 2011). Conforme demonstrado, os resultados obtidos sobre Karate-Dō correspondem a esses achados da literatura, permitindo que possa ser configurado como uma possível ferramenta de treinamento cognitivo de similar eficácia.

Apesar de não ser o foco deste estudo, é importante ressaltar que o exercício físico pode estimular certos mecanismos biofisiológicos, como aumento do fator neurotrófico de crescimento neural (Cotman \& Engesser-Cesar, 2002) e alterações no metabolismo encefálico (Eggermont, Swaab, Luiten \& Scherder, 2006), podendo influenciar nos resultados apresentados. Além disso, o convívio social proporcionado pode agir em conjunto com as alterações neurofisiológicas, potencializando a melhora de sintomas cognitivos e comportamentais (Vance, Wadley, Ball, Roenker \& Rizzo, 2005); diversos estudos demonstraram que o engajamento social (como fazer trabalho voluntário, participar de clubes, ir à igreja), participação em redes sociais e maior histórico de contato social estão associadas à redução do risco de declínio cognitivo (Noice, Noice \& Kramer, 2014; Brown et al., 2012; James, Wilson, Barnes \& Bennett, 2011; Seeman et al., 2011; Ertel, Glymour \& Berkman, 2008; Crooks, Lubben, Petitti, Little \& Chiu, 2008) e baixa participação social, contato social menos frequente e solidão são fatores estatisticamente significativos para a incidência de problemas cognitivos (Kuiper et al., 2015).

O presente estudo, no entanto, apresenta algumas limitações. Um dos pontos de maior dificuldade foi a obtenção de uma amostra que atendesse a todos os critérios de inclusão, além da manutenção da quantidade de participantes até o final do protocolo de intervenção. Trata-se, também, de uma amostra pequena, de conveniência, e sem comparação com grupo controle, o que não permite a generalização dos dados. E, conforme salienta Etnier et al. (1997), períodos mais prolongados de treinamento, desde que focados e objetivos, podem produzir efeitos de maior impacto do que os realizados de forma aguda, favorecendo o desempenho cognitivo. Contudo, ressalvadas as limitações, este é um estudo pioneiro ao investigar os efeitos de um treinamento de Karate-Dō, estilo Wadō-ryū, sobre a cognição de idosos com transtorno neurocognitivo leve.

\section{Conclusão}

Os resultados deste trabalho sugerem que a prática de três meses de Karate-Dō, estilo Wadō-ryū, está relacionada a uma melhora significativa da cognição em idosos diagnosticados com transtorno neurocognitivo leve. Foi possível constatar melhoras significativas quanto às tarefas de memória visual (evocação), atenção, atenção alternada, memória de trabalho (visual e verbal), memória verbal episódica (evocação imediata) e funções executivas (capacidade de raciocínio lógico-abstrato e velocidade de processamento visual).

Mesmo a amostra sendo pequena, o que não permite generalizações dos resultados, pode-se evidenciar que o Karate-

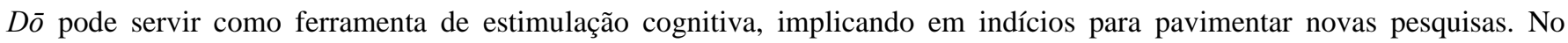
entanto, os dados são promissores e justificam maiores investigações. É indicado que estudos futuros elaborem protocolos de ensaios clínicos randomizados cegos, com amostras maiores, períodos de treinamento prolongados e/ou de maior frequência 
semanal, além da realização de um acompanhamento de longo prazo.

\section{Agradecimentos}

O presente trabalho foi realizado com apoio da Coordenação de Aperfeiçoamento de Pessoal de Nível Superior Brasil (CAPES) - Código de Financiamento 001.

\section{Referências}

Army Individual Test Battery. (1944). Manual of directions and scoring. War Department, Adjutant General's Office.

Almeida, O. P. \& Almeida, S. A. (1999). Confiabilidade da versão brasileira da Escala de Depressão em Geriatria (GDS) versão reduzida. Arquivos de Neuropsiquiatria, 57(2B), 421-426.

Antunes, H. K. M., Santos, R. F., Heredia, R. A. G., Bueno, O. F. A. \& Mello, M. T. (2001). Alterações Cognitivas em Idosas Decorrentes do Exercício Físico Sistematizado. Revista da Sobama, 6(1), 27-33.

American Psychiatric Association. (2014). DSM-5: Manual Diagnóstico e Estatístico de Transtornos Mentais. Artmed.

Brown, C. L., Gibbons, L. E., Kennison, R. F., Robitaille, A., Lindwall, M., Mitchell, M. B., Shirk, S. D., Atri, A., Cimino, C. R., Benitez, A., Macdonald, S. W. S., Zelinski, E. M., Willis, S. L., Schaie, K. W., Johansson, B., Dixon, R. A., Mungas, D. M., Hofer, S. M. \& Piccinin, A. M. (2012). Social activity and cognitive functioning over time: a coordinated analysis of four longitudinal studies. Journal of Aging Research, 12(0), Article ID 287438.

Burke, D. T., Al-Adawi, S., Lee, Y. T. \& Audette, J. (2007). Martial Arts as Sport and Therapy. The Journal of Sports Medicine and Physical Fitness, 47(1), 96-102.

Chang, J. Y., Tsai, P., Beck, C., Hagen, J., Huff, D. C., Anand, K. J. S., Roberson, P. K., Rosengren, K. \& Beuscher, L. (2011). The Effect of Tai Chi on Cognition in Elders with Cognitive Impairment. MEDSURG Nursing, 20(2), 63-70.

Chaves, M. L. \& Izquierdo, I. (1992). Differential diagnosis between dementia and depression: a study of efficiency increment. Acta Neurol Scand, 85(6), 378382.

Conant, K. D., Morgan, A. K., Muzykewicz, D., Clark, D. C. \& Thiele, E. A. (2008). A Karate Program for Improving Self-Concept and Quality of Life in Childhood Epilepsy: Results of a pilot study. Epilepsy \& Behavior, 12(0) 61-65.

Cotman, C. W. \& Engesser-Cesar, C. (2002). Exercise enhances and protects brain function. Exercise and Sport Sciences Reviews, 30(2), 75-79.

Crooks, V. C., Lubben, J., Petitti, D. B., Little, D. \& Chiu, V. (2008). Social Network, Cognitive Function, and Dementia Incidence Among Elderly Women. American Journal of Public Health, 98(7), 1221-1227.

Cunha, J. A., Trentini, C. M., Argimon, I. L., Oliveira, M. S., Werlang, B. G. \& Prieb, R. G. (2005). Teste Wisconsin de Classificação de Cartas: manual. São Paulo: Casa do Psicólogo.

Diamond, A. \& Lee, K. (2011). Interventions Shown to Aid Executive Function Development in Children 4 to 12 Years Old. Science, 333(6045), 959-964.

Eggermont, L., Swaab, D., Luiten, P. \& Scherder, E. (2006). Exercise, Cognition and Alzheimer's Disease: more is not necessarily better. Neuroscience \& Biobehavioral Reviews, 30(4), 652-675.

Ertel, K. A., Glymour, M. M. \& Berkman, L. F. (2008). Effects of Social Integration on Preserving Memory Function in a Nationally Representative US Elderly Population. American Journal of Public Health, 98(7), 1215-1220.

Etnier, J. L., Salazar, W., Landers, D. M., Petruzzello, S. J., Han, M. \& Nowell, P. (1997). The influence of physical fitness and exercise upon cognitive functioning: a meta-analysis. Journal of Sport \& Exercise Psychology, 19(0), 249-74.

Frederiksen, K. S., Verdelho, A., Madureira, S., Bäzner, H., O'Brien, J. T., Fazekas, F., Scheltens, P., Schmidt, R., Wallin, A., Wahlund, L. O., Erkinjunttii, T., Poggesi, A., Pantoni, L., Inzitari, D. \& Waldemar, G. (2015). Physical activity in the elderly is associated with improved executive function and processing speed: the LADIS Study. International journal of geriatric psychiatry, 30(7), 744-750.

Funakoshi, G. (1973). Karate-Dō Kyōhan: The master text. Tóquio: Kodansha International.

Greenhalgh, T. (2005). Como Ler Artigos Científicos: Fundamentos da medicina baseada em evidências. Artmed.

Hillman, C. H., Pontifex, M. B., Castelli, D. M., Khan, N. A., Raine, L. B., Scudder, M. R., Drollette, E.S., Moore, R. D., Wu, C. T. \& Kamijo, K. (2014). Effects of the FITKids randomized controlled trial on executive control and brain function. Pediatrics, 134(4), e1063-71.

Hochman, B., Nahas, F. X., Oliveira Filho, R. S. \& Ferreira, L. M. (2005). Desenhos de pesquisa. Acta Cirúrgica Brasileira, 20 (supl. 2), $2-9$.

Jäger, K., Schmidt, M., Conzelmann, A. \& Roebers, C. M. (2015). The effects of qualitatively different acute physical activity interventions in real-world settings on executive functions in preadolescent children. Mental Health and Physical Activity, 9(0), 1-9.

James, B. D., Wilson, R. S., Barnes, L. L. \& Bennett, D. A. (2011). Late-life social activity and cognitive decline in old age. Journal of the International Neuropsychological Society, 17(6), 998-1105. 
Jansen, P. \& Dahmen-Zimmer, K. (2012). Effects of Cognitive, Motor, and Karate Training on Cognitive Functioning and Emotional Well-Being of Elderly People. Frontiers of Psychology, 3(40), 1-7.

Kasai, J. Y. T., Busse, A. L., Magaldi, R. M., Soci, M. A., Rosa, P. M., Curiati, J. A. E. \& Jacob Filho, W. (2010). Effects of Tai Chi Chuan on cognition of elderly women with mild cognitive impairment. Einstein, 8(1), 40-45.

Kuiper, J. S., Zuidersma, M., Voshaar, R. C. O., Zuidema, S. U., Heuvel, E. R., STOLK, R. P. \& Smidt, N. (2015). Social relationships and risk of dementia: A systematic review and meta-analysis of longitudinal cohort studies. Ageing Research Reviews, 22(0), 39-57.

La Rue, A. (2010). Healthy brain aging: Role of cognitive reserve, cognitive stimulation, and cognitive exercises. Clinics in Geriatric Medicine, 26(1), 99-111.

Lam, L. C. W., Chau, R. C. M., Wong, B. M. L., Fung, A. W. T., Lui, V. W. C., Tam, C. C. W., Leung, G. T. Y., Kwok, T. C. Y., Chiu, H. F. K., Ng, S. \& Chan, W. M. (2011). Interim follow-up of a randomized controlled trial comparing Chinese style mind body (Tai Chi) and stretching exercises on cognitive function in subjects at risk of progressive cognitive decline. International journal of geriatric psychiatry, 26(0), 733-740.

Lima, A. L. S. (2014). Efeito do exercício físico sobre a cognição de idosos com comprometimento cognitivo leve. Monografia de Graduação em Fisioterapia, Universidade Estadual da Paraíba, Campina Grande. PB, Brasil. Recuperado em português 01, junho, 2021, de https://cutt.ly/angQCUE.

Lima, R. F., Silva, V. F., Oliveira, G. L., Oliveira, T. A. P., Fernandes Filho, J., Mendonça, J. G. R., Borges, C. J.; Militão, A. G., Freire, I. Q. \& Silva, J. R. V. (2017). Practicing karate may improves executive functions of 8-11-year-old schoolchildren. Journal of Physical Education and Sport,17 (4), $2513-2518$.

Lopes Filho, B. J. P. (2015). Efeitos do Treinamento de Karate-Dō na Cognição de Idosos: Ensaio Clínico Randomizado e Controlado. Dissertação de Mestrado, Pontifícia Universidade Católica do Rio Grande do Sul, Porto Alegre. RS, Brasil. https://repositorio.pucrs.br/dspace/handle/10923/7644.

Lopes Filho, B. J. P., Oliveira, C. R. \& Gottlieb, M. G. V. (2019). Effects of Karate-Do Training in Older Adults Cognition: Randomized controlled trial. Journal of Physical Education, 30(1), e3030.

Malloy-Diniz, L. F., Lasmar, V. A. P., Gazinelli, L. S. R., Fuentes, D. \& Salgado, J. V. (2007). The Rey Auditory-Verbal Learning Test: Applicability for the Brazilian elderly population. Revista Brasileira de Psiquiatria, 29(4), 324-329.

Man, D. W. K., Tsang, W. W. N. \& Hui-Chan, C. W. Y. (2010). Do Older T'ai Chi Practitioners Have Better Attention and Memory Function? Journal of alternative and complementary medicine, 16(12), 1259-1264.

Nascimento, E. (2004). WAIS III - Escala de inteligência Wechsler para adultos. Casa do Psicólogo.

Nakayama, M. (2000a). O Melhor do Karatê: Visão Abrangente - Práticas. Cultrix.

Nakayama, M. (2000b). O Melhor do Karatê: Fundamentos. Cultrix.

Nakayama, M. (2000c). O Melhor do Karatê: Heian e Tekki. Cultrix.

Nakayama, M. (2000d). O Melhor do Karatê: Kumite 1. Cultrix.

Nguyen, M. H. \& Kruse, A. (2012). A randomized controlled trial of Tai chi for balance, sleep quality and cognitive performance in elderly Vietnamese. Clinical interventions in aging, 7(0), 185-90.

Nitrini, R., Caramelli, P., Herrera Jr., E., Charchat-Fichman, H. \& Porto, C.S. (2005). Performance in Luria’s Test According to Educational Level. Cognitive and Behavioral Neurology, 18(4), 211-214.

Noice, T., Noice, H. \& Kramer, A. F. (2014). Participatory arts for older adults: a review of benefits and challenges. Gerontologist, 54(5), 741-753.

Northey, J. M., Cherbuin, N., Pumpa, K. L., Smee, D. J. \& Rattray, B. (2018). Exercise interventions for cognitive function in adults older than 50: a systematic review with meta-analysis. British Journal of Sports Medicine, 52(3), 154-160.

Nucci, M., Mapelli, D., \& Mondini, S. (2012). Cognitive Reserve Index questionnaire (CRIq): A new instrument for measuring cognitive reserve. Aging Clinical and Experimental Research, 24(3), 218-226.

Nunan, D. (2006). Development of a Sports Specific Aerobic Capacity Test for Karate: a Pilot Study. Journal of Sports Science \& Medicine, 5(0), 47-53.

Oliveira, M. S. \& Rigoni, M. S. (2010). Figuras Complexas de Rey. Casa do Psicólogo.

Proust-Lima, C., Amieva, H., Letenneur, L., Orgogozo, J., Jacqmingadda, H. \& Dartigues, J. (2008). Gender and Education Impact on Brain Aging: A General Cognitive Factor Approach. Psychology and Aging, 23(3), 608-620.

Rami, L., Valls-Pedret, C., Bartrés-Faz, D., Caprile, C., Solé-Padullés, C., Castellvi, M., Olives, J., Bosch, B., \& Molinuevo, J. L. (2011). Cognitive reserve questionnaire. Scores obtained in a healthy elderly population and in one with Alzheimer's disease. Revista de Neurología, 52(4), 195-201.

Rios, S. O., Marks, J., Estevan, I. \& Barnett, L. M. (2017). Health benefits of hard martial arts in adults: a systematic review. Journal of Sports Sciences, 36(14), 1614-1622.

Salthouse, T. A. (2014). Frequent assessments may obscure cognitive decline. Psychological Assessment, 26(4), 1063-1069.

Schmidt, R.A. \& LEE, T.D. (2013). Motor Learning and Performance: from principles to application. Human Kinetics Publisher.

Seeman, T. E., Miller-Martinez, D. M., Stein Merkin, S., Lachman, M. E., Tun, P. A. \& Karlamangla, A. S. (2011). Histories of social engagement and adult cognition: Midlife in the U.S. study. The Journals of Gerontology Series B: Psychological Sciences and Social Sciences, 66B (s1), 141-152. 
Research, Society and Development, v. 10, n. 8, e6310817100, 2021

(CC BY 4.0) | ISSN 2525-3409 | DOI: http://dx.doi.org/10.33448/rsd-v10i8.17100

Stern, Y. (2012). Cognitive reserve in ageing and Alzheimer's disease. Lancet Neurology, 11(11), 1006-1012.

Sternberg, R. J. (2008). Psicologia Cognitiva. Artmed.

Strauss, E., Sherman, E. M. S. \& Spreen, O. (2006). A Compendium of Neuropsychological Tests: Administration, Norms and Commentary. Oxford University Press.

Vance, D., Wadley, V., Ball, K., Roenker, D. \& Rizzo, M. (2005). The effects of physical activity and sedentary behavior on cognitive health in older people. Journal of Aging and Physical Activity, 13(3), 294-313.

Wechsler, D. (1987). Wechsler Memory Scale-Revised Manual. Psychological Corporation. 